Case report

\title{
Trichosporon asahii late onset sepsis in an extremely low birth weight infant
}

\author{
Maria Catalina Vaz Ferreira*, Juan Pablo Gesuele \\ Department of Neonatology, Pereira Rossell Children's Hospital, Montevideo, Uruguay
}

\begin{abstract}
We describe a case of Trichosporon asahii late-onset sepsis in an extremely low birth weight infant from our neonatal intensive care unit. The baby presented early neutropenia and received therapy with granulocyte colony-stimulating factor. At day of life 8 he showed clinical signs of sepsis. Forty-eight hours after beginning of symptoms, blood culture developed $T$. asahii. Baby responded to treatment with liposomal amphotericin B. The aim of this report is to discuss a case of late onset sepsis in an immuno-compromised patient due to an unusual pathogen, with variable or intrinsic resistance to the most commonly used antifungal therapies. Appropriate use of antifungal in this vulnerable population is of pivotal importance for both treatment and prevention of infection.

Keywords: late onset sepsis; Trichosporon asahii; extremely low birth weight infant
\end{abstract}

\section{Introduction}

Despite being part of the normal skin flora, Trichosporon asahii is an unusual pathogen in neonates. It is found to be the second most common causative agent of infection in immuno-deficient adult population [1]. In neonates, reports estimate a rate of $0.36 \%$ of fungemia secondary to Trichosporon [2], reaching a mortality rate as high as $80 \%$ [3]. Our patient was found to have several risk factors for Trichosporon asahii sepsis, such as severe and early neutropenia, extremely low birth weight, male, prolonged use of vascularaccess catheters and neonatal intensive care unit (NICU) stay [4].

Trichosporon asahii species are noncapsulated yeasts. Widely distributed in the environment, Trichosporon asahii is part of the normal skin flora of the perineal region and upper respiratory tract and gut microbiota [5]. From the 37 described species, only 16 have been associated with bloodstream infections

Received: February 2018; Accepted after review: March 2018; Published: March 2018.

${ }^{*}$ Corresponding author: Maria Catalina Vaz Ferreira, MD, Department of Neonatology, Pereira Rossell Children's Hospital, University of the Republic, Montevideo, Uruguay.

Email: catalinavazferreira@gmail.com and there has been a boost in the overall incidence in immuno-compromised patients [4]. The vast majority correspond to $T$. asahii (74\%), followed by $T$. dermatis (12\%) [4].

They represent the second most common cause of fungemia in hemato-oncological patients. Mortality associated with invasive fungemia secondary to Trichosporon in immune-compromised patients ranges from 55 to $80 \%$ [4], however, there are no mortality reports in neonates. Early instauration of appropriate antifungal treatment may increase survival. These yeast species show high resistance to Amphotericin and Echinocandins, therefore, prognosis is poor [1].

\section{Case Report}

A 26 weeker male infant was born to a G2P2, 27-year-old mother. Pregnancy was well controlled. Mother was admitted at 26 weeks of gestation and delivery was via CSection due to severe PreeclampsiaEclampsia syndrome with altered Doppler signal. Magnesium sulfate and a full course of prenatal corticosteroids were administered. Rupture of membranes was during C-section 
with clear amniotic fluid. Cord clamp was delayed, and cord blood gases showed no acidosis.

Baby weighed 850 grams, APGAR scores were $7 / 8$, he was admitted to the NICU and placed on mechanical ventilation due to respiratory distress syndrome (RDS) and umbilical catheters were placed. Trophic feeds were started at day of life (DOL) 1 and prophylactic antifungal therapy started with Fluconazole.

Baby developed severe neutropenia at DOL 2, with absolute polymorphonuclear count $<500 \mu / L$, and was started with granulocyte colony-stimulating factor. Due to favorable clinical evolution in addition to negative blood culture, Ampicillin-Gentamicin were discontinued at DOL 5 . At that time, baby continued to be ventilated and received a red blood cell (RBC) transfusion given a hematocrit (Hct) of $27 \%$. Transfontanelar ultrasound showed no active bleeding.

At DOL 8, baby physical exam revealed a tense, distended and blue colored abdomen. Serum sodium was $119 \mathrm{mmol} / \mathrm{L}$, CRP $89 \mathrm{mg} / \mathrm{L}$ and platelet count 14000/L. With the presumptive diagnosis of late onset sepsis, baby was started empirically on antibiotic treatment with Vancomycin- Meropenem and Fluconazole changed to treatment dose.

Preliminary blood culture results showed fungal proliferation, consequently antibiotics were discontinued and Fluconazole changed to Amphotericin B. Fungal cultures and microscopic mycological analysis confirmed Trichosporon asahii, therefore, 21 days of treatment after blood culture negativation were completed with liposomal Amphotericin B. Susceptibility assays were not performed. Two intra-treatment and one after treatment blood cultures were performed, all of them were negative. Baby's clinical status improved significantly, at DOL 16 he was extubated. Renal function and abdomen ultrasound were normal. Pathology showed intrervillous thrombosis and early placental maturation.

\section{Discussions}

In neonates, late onset sepsis secondary to Trichosporon asahii is an unusual condition.
Rare opportunist yeast infections occur mainly in susceptible hosts. Patients with specific risk factors are prone to develop this infrequent infection. In such population some reports estimate $0.36 \%$ of the cases of fungemia to Trichosporon species, with high mortality [2]. In this case, our patient was male, had a long stay at the NICU, had central catheters, showed early onset neutropenia requiring treatment and received prophylactic treatment with Fluconazole as part of our NICUs protocol. All these facts have been associated with the development of invasive infections to Trichosporon, being fungemia the most frequent way of manifestation.

An undamaged immune system is essential in opportunistic infections control. The pathogen-host interaction is crucial in the pathogenesis of invasive fungal infections. A defective or decontrolled host immune response will determine in most cases the severity and results of invasive fungemia. Although absolute neutrophil count (innate immunity) play a major role as first line host barrier, cellular immunity (adaptative) is de main host defensive mechanism against fungemia. The balance between the immune $L$ Th1 and $L$ Th2 mediated response is the final determinant of the severity and prognosis in the immune compromised patients undergoing fungemia.

Granulocyte colony-stimulating factors foster development and maturation of granulocyte colonies. There are controversies regarding the role that plays the use of granulocyte colony-stimulating factor in patients with severe neutropenia. A 2003 Cochrane review demonstrated a faster increase in the total neutrophil count, however, no changes were observed in short term mortality $[6,7]$.

The early identification of risk factors for fungemia in a preterm baby is clue when dealing with patients that show a sudden deterioration of their clinical status. Appropriate election of antifungal therapy is challenging and should be done according to local epidemiology. Candidemia is the main cause of fungal sepsis, therefore, preferred prophylactic antifungal therapy is commonly done with first generation Azoles such as Fluconazol and Polyenes (Amphotericin B), 
both with adequate penetration in central nervous system (CNS) and renal parenchyma.

Trichosporon asahii shows in vitro resistance to Amphotericin B with MICs $\geq 2$ $\mathrm{mg} / \mathrm{L}$ and Caspofungin resistance with MICs $\geq 16 \mathrm{mg} / \mathrm{L}[1,4,8]$. For this reason, Amphotericin B should not be choose as first line therapy in immune-compromised patients with fungemia. In our country, we do not have de possibility to assess the MIC of the pathogens on regular clinical practice.

In vitro studies suggest Azoles, especially Voriconazole, have the best efficacy profile against all species of Trichosporon [1]. Most pharmacokinetic studies in children recommend a dose of $9 \mathrm{mg} / \mathrm{kg} /$ dose every 12 hours with an excellent penetration to the CNS [9]. The restoration of immune function is of great importance in the final prognosis, together with appropriate antifungal treatment. Controversies arise when considering routine use of granulocyte colony-stimulating factor to boost the immune response.

\section{References}

1. Miceli $\mathrm{MH}$, Díaz JA, Lee SA. Emerging opportunistic yeast infections. Lancet Infect Dis 2011; 11(2):142-151.

2. Basu S, Tilak R, Kumar A. Multidrug-resistant Trichosporon: an unusual fungal sepsis in preterm neonates. Pathog Glob Health 2015; 109(4):202-206.

3. Ozkaya-Parlakay A, Karadag-Oncel E, Cengiz $\mathrm{AB}$, et al. Trichosporon asahii sepsis in a patient with pediatric malignancy. $J$ Microbiol Immunol Infect 2016; 49(1):146-149.

4. Arendrup $\mathrm{MC}$, Boekhout $\mathrm{T}$, Akova $\mathrm{M}$, et al. ESCMID and ECMM joint clinical guidelines for the diagnosis and management of rare invasive yeast infections. Clin Microbiol Infect 2014; 20(Suppl. 3):76-98.

5. Alzate Atehortuaa MA, Garzón J, Valderrama S, Zhong A. Tricosporonosis en un paciente con leucemia aguda. Acta Colomb Cuid Intensivo 2017; 17(1):68-75.

\section{Conclusions}

Although infrequent, Trichosporon asahii late onset sepsis in neonates has a high mortality and represents a therapeutic challenge for clinicians due to the pathogen's variety in its response to the most commonly used antifungal agents.

Some of the most important predisposing factors of Trichosporon late onset sepsis are: a deficient immune system, together with a prolonged stay at the NICU, presence of venous catheters, prematurity and very low birth weight. Early treatment of neutropenia with granulocyte colony-stimulating factor might play an important role in the rapid increase of neutrophil count with no changes in short term mortality.

\section{Consent}

Written informed consent was obtained from the patient's parents for publication of this case report.

\section{Conflict of interest}

The author(s) declare that they have no competing interests.

6. Castagnola E, Dufour C. Role of G-CSF and GM-CSF in the management of infections in preterm newborns: an update. Early Hum Dev 2014; 90(Suppl. 2):S15-S17.

7. Aktas D, Demirel B, Gursoy T, Ovali F. A randomized case-controlled study of recombinant human granulocyte colony stimulating factor for the treatment of sepsis in preterm neutropenic infants. Pediatr Neonatol 2015; 56(3):171-175.

8. Chitasombat $\mathrm{MN}$, Kofteridis $\mathrm{DP}$, Jiang $\mathrm{Y}$, Tarrand J, Lewis RE, Kontoyiannis DP. Rare opportunistic (non-Candida, non-Cryptococcus) yeast bloodstream infections in patients with cancer. J Infect 2012; 64(1):68-75.

9. Puia-Dumitrescu M, Smith PB. Antifungal drugs in newborns and children. Pediatr Clin N Am 2017; 64(6):1389-1402. 